\title{
Photoemission study of praseodymia in its highest oxidation state: The necessity of in situ plasma treatment
}

\author{
A. Schaefer, ${ }^{1}$ S. Gevers, ${ }^{2}$ V. Zielasek,,${ }^{1, a)}$ T. Schroeder, ${ }^{3}$ J. Falta, ${ }^{4}$ \\ J. Wollschläger, ${ }^{2}$ and $\mathrm{M}$. Bäumer ${ }^{1}$ \\ ${ }^{1}$ Institute of Applied and Physical Chemistry, University of Bremen, Leobener Str. NW2, D-28359 Bremen, \\ Germany \\ ${ }^{2}$ Department of Physics, University of Osnabrück, Barbarastr. 7, D-49069 Osnabrück, Germany \\ ${ }^{3}$ IHP, Im Technologiepark 25, D-15236 Frankfurt (Oder), Germany \\ ${ }^{4}$ Institute of Solid State Physics, University of Bremen, P.O. Box 330440, D-28334 Bremen, Germany
}

(Received 16 August 2010; accepted 26 October 2010; published online 1 February 2011)

\begin{abstract}
A cold radio frequency oxygen plasma treatment is demonstrated as a successful route to prepare clean, well-ordered, and stoichiometric $\mathrm{PrO}_{2}$ layers on silicon. High structural quality of these layers is shown by $\mathrm{x}$-ray diffraction. So far unobserved spectral characteristics in $\operatorname{Pr} 3 d \mathrm{x}$-ray photoelectron (XP) spectra of $\mathrm{PrO}_{2}$ are presented as a fingerprint for praseodymia in its highest oxidized state. They provide insight in the electronic ground state and the special role of praseodymia among the rare earth oxides. They also reveal that former XP studies suffered from a significant reduction at the surface. (C) 2011 American Institute of Physics. [doi:10.1063/1.3516953]
\end{abstract}

\section{INTRODUCTION}

The oxides of rare earth (RE) metals are highly interesting and are intensively studied materials because of their special electronic structures, which originate from the narrow $f$ bands of the respective metal and the small overlap of these bands with the oxygen $p$ orbital-derived valence band. Strong electron-electron correlation effects lead to a high degree of localization of the $f$ electrons. Since the energy differences between different $f$ configurations are small, mixed-valence states of the cations are possible which are the prerequisite for nonstoichiometric compounds. Due to the concomitant formation of oxygen vacancies with high mobility and their ordering, such oxides can show a wealth of different crystal structures, which may display very different electrical and magnetic properties. ${ }^{1}$

The high degree of screening and localization of the metal $f$ electrons in rare earth oxides (REOs) leads, in general, to a high similarity of the REO properties as the atomic number is varied. Therefore, it is surprising that there are a few exceptions not fitting in the overall trends within the row of the REOs: All rare earth metals form sesquioxides $\left(\mathrm{RE}_{2} \mathrm{O}_{3}\right)$, e.g., while dioxides $\left(\mathrm{REO}_{2}\right)$ are only formed by cerium, praseodymium, and terbium. Among these elements, praseodymia is furthermore distinguished, as it exhibits by far the highest oxygen mobility in the form of $\operatorname{Pr}_{6} \mathrm{O}_{11},{ }^{2}$ which is the stable stoichiometry under atmospheric conditions up to $300-400{ }^{\circ} \mathrm{C}$. Apart from $\operatorname{Pr}_{6} \mathrm{O}_{11}$, a rich phase diagram exists with a large number of phases between $\mathrm{Pr}_{2} \mathrm{O}_{3}$ and $\mathrm{PrO}_{2}$. These differ only slightly in stoichiometry $\left(\mathrm{Pr}_{n} \mathrm{O}_{2 n-2}\right.$ with $n=4,7,9,10,11$, or 12 is known), and allow the system to give off or incorporate oxygen easily in the crystal lattice. As a consequence, $n$ - and $p$-semiconducting properties and addi-

\footnotetext{
a) Author to whom correspondence should be addressed. Electronic mail: zielasek@uni-bremen.de. Tel.: +49 421218 63180. Fax.: +49 421218 63188.
}

tional ionic conductivity at higher temperatures ${ }^{3}$ are observed. The remarkable crystallographic as well as electronic flexibility render praseodymia highly interesting for microelectronic ${ }^{4}$ as well as surface chemical applications, such as sensors ${ }^{5}$ or catalysis. $^{6}$

An optimization of the properties and a targeted use of such multivalent oxides requires an in-depth understanding of the electronic structure. For this purpose, electron spectroscopies working under ultrahigh vacuum (UHV) conditions are intensively used techniques. Aiming, however, at studying the whole range of oxidation states bears the risk that the highly oxidized states of the oxide sample are inaccessible because any sample preparation involving thermal treatment will result in a loss of oxygen. In the worst case, only a partially reduced state can be studied.

In the present work, we will show that cold radio frequency (RF) plasmas can be used to selectively prepare highly oxidized, single crystalline films, such as $\mathrm{PrO}_{2}$, which are very difficult or even impossible to obtain by conventional methods. As a result, we observe new spectral characteristics in the $\mathrm{Pr} 3 d$ core level spectra of the clean $\mathrm{PrO}_{2}$ surface which have not been reported before. They point to a population of the $\operatorname{Pr} 4 f$ states being higher than values proposed by previous theoretical reports, and shed new light on the special role of praseodymia in the REO series.

\section{EXPERIMENTAL}

The $\mathrm{PrO}_{2} / \mathrm{Si}(111)$ samples for this study were prepared by first growing hexagonal $\mathrm{Pr}_{2} \mathrm{O}_{3}$ on a silicon(111) substrate by molecular beam epitaxy. ${ }^{7}$ Subsequently, the samples were annealed $e x$ situ to $450{ }^{\circ} \mathrm{C}$ in oxygen atmosphere $\left(p=10^{5} \mathrm{~Pa}\right)$ in order to form cubic $\mathrm{PrO}_{2}$. These films exhibit (111) orientation and exclusive type-B stacking ${ }^{8}$ and have a total thickness of $\sim 15 \mathrm{~nm}$. For the oxygen plasma treatment, a specially designed UHV-compatible, capacitively coupled RF plasma 


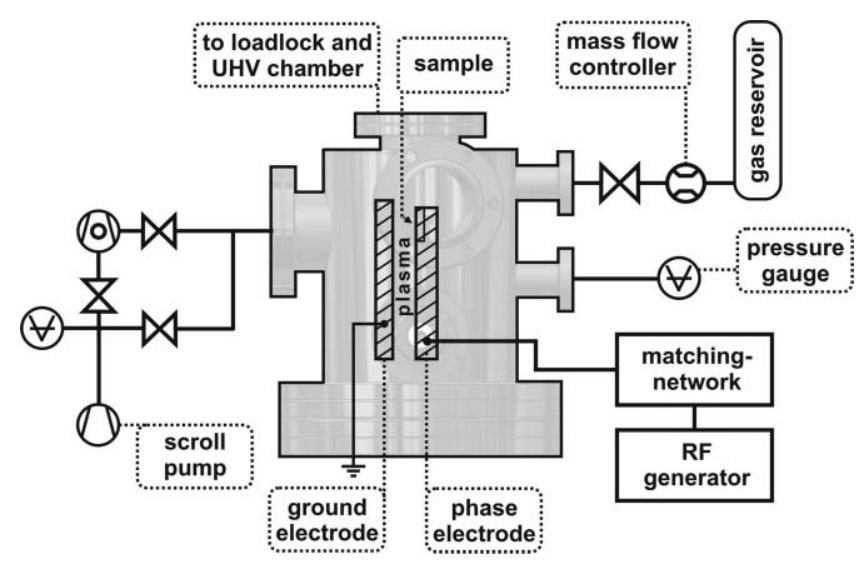

FIG. 1. Schematic of the capacitively coupled plasma source, including pumps, gas supply, and power lines. The sample is inserted directly into the phase electrode so that the sample surface is homogeneously exposed to plasma. From the plasma source the sample can be directly transferred into a UHV chamber with TPD, LEED, and XPS.

source was used (Fig. 1). ${ }^{9}$ The sample was integrated in the phase electrode and its surface directly exposed to the plasma for $60 \mathrm{~min}$ at moderate power and under continuous gas flow. After plasma treatment the samples were transferred into the UHV chamber for XPS measurements without breaking the vacuum. For XPS nonmonochromatized $\mathrm{Al} \mathrm{K} \alpha$ radiation was used for photoelectron generation and a Leybold E100 hemispherical energy analyzer with a one-channeltron detector for analysis. Low-energy electron diffraction (LEED) was performed using an Omicron SPECTALEED. Temperature programmed desorption (TPD) spectra were recorded by heating the sample with a rate of $1 \mathrm{~K} / \mathrm{s}$ in front of a differentially pumped quadrupole mass spectrometer (Hiden Analytical).

For x-ray diffraction (XRD) measurements the samples were transported under a clean oxygen atmosphere to HASYLAB beamlines BW2 and W1 at the DESY radiation source, mounted to a six-circle diffractometer, and irradiated by $10 \mathrm{keV}$ photons. The diffracted intensity was detected by a linear silicon detector.

\section{RESULTS}

In this section, an XPS analysis of the effects of oxygen plasma on the chemical composition of the oxide film and its surface will be presented. As a main part a detailed discussion of the praseodymium $3 d$ spectra and the features visible therein will be provided. The XPS results will then be supplemented by TPD and a structure analysis based on the XRD data obtained from the untreated and the plasma-treated film. Additionally, results of a film exposed to atmosphere again after the oxygen plasma treatment will be discussed.

\section{A. XPS analysis}

Figure 2(a) shows XP spectra in the energy range of the carbon $1 \mathrm{~s}$ emission before (i) and after (ii) oxygen plasma treatment of the praseodymia film. The spectrum (i) clearly reveals a large amount of carbon contamination on the oxide surface caused by exposure to the atmosphere. Addition-

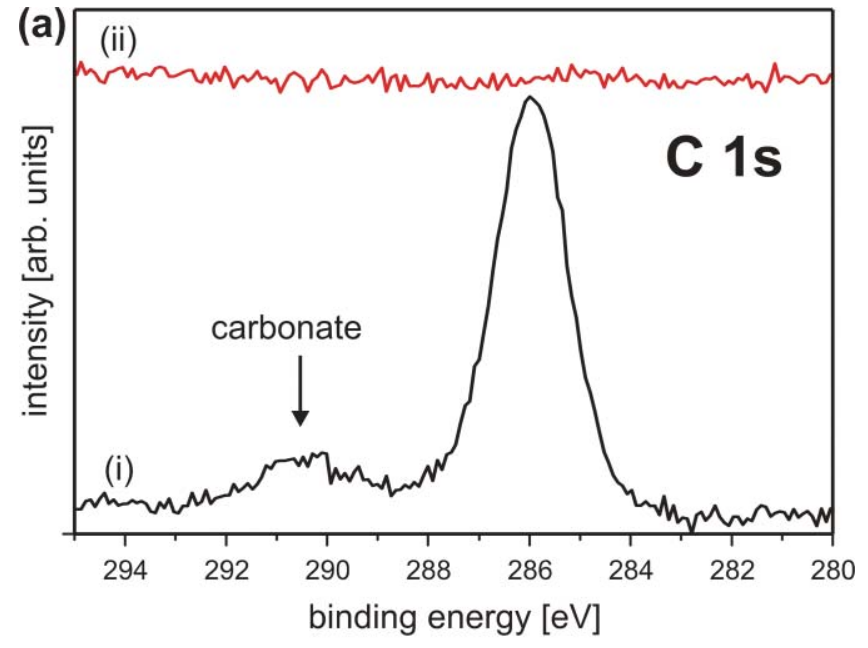

(b)

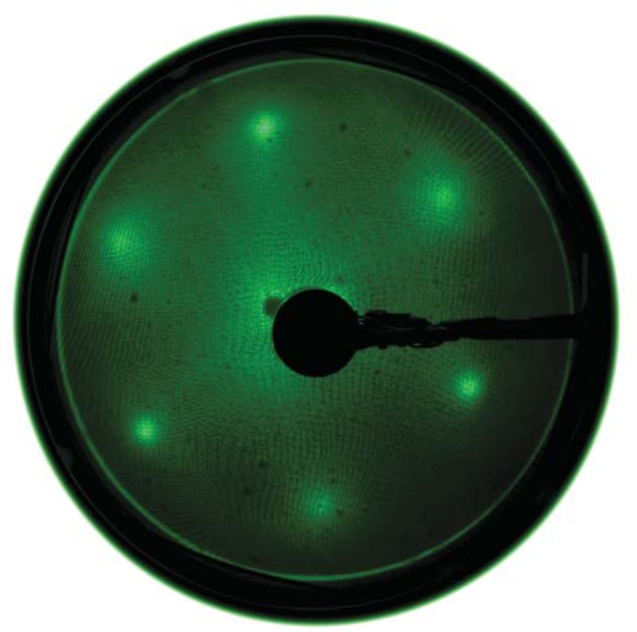

FIG. 2. (a) XP spectra of the carbon 1s level of the (i) untreated and (ii) plasma-treated sample. The untreated sample exhibits a large amount of carbon on the surface along with carbonate formed from reaction with atmospheric $\mathrm{CO}_{2}$. After exposing the surface to oxygen plasma the carbon content is below the XPS detection limit. (b) LEED image recorded after oxygen plasma treatment. Clearly a sharp $1 \times 1$ diffraction pattern of the $\mathrm{PrO}_{2}(111)$ surface is observable which was impossible to achieve by other cleaning methods.

ally, a small signal at $\sim 290.5 \mathrm{eV}$ indicates carbonate species from reaction of the oxide surface with atmospheric $\mathrm{CO}_{2}$. As shown by spectrum (ii), the carbon species on the surface can be completely removed by subjecting the film to the oxygen plasma. After the treatment of the film with oxygen plasma, even a sharp $(1 \times 1)$ LEED-pattern of the $\mathrm{PrO}_{2}$ was obtained [Fig. 2(b)] which was impossible to achieve by common cleaning methods in ultrahigh vacuum, such as annealing at elevated oxygen pressures in front of a gas doser (up to $10^{-6}$ mbar oxygen background pressure).

Several groups have already reported on XPS studies of surfaces of Pr dioxide. ${ }^{10-14}$ Spectrum (a) in Fig. 3 shows a typical $\mathrm{Pr} 3 d$ spectrum of an ex situ prepared $\mathrm{PrO}_{2}$ layer on $\mathrm{Si}(111)$ that we obtained in our own experiments. It very much resembles previous spectra from pressed $\mathrm{PrO}_{2}$ powders, ${ }^{10,13}$ on which calculations of Kotani and others were based. ${ }^{15}$ The multitude of peaks in the $\operatorname{Pr} 3 d$ spectrum of $\mathrm{PrO}_{2}$ has been ascribed to a mixed 3+/4+ valency of Pr. Provided that pure $\mathrm{PrO}_{2}$ and not partially reduced material is studied, 


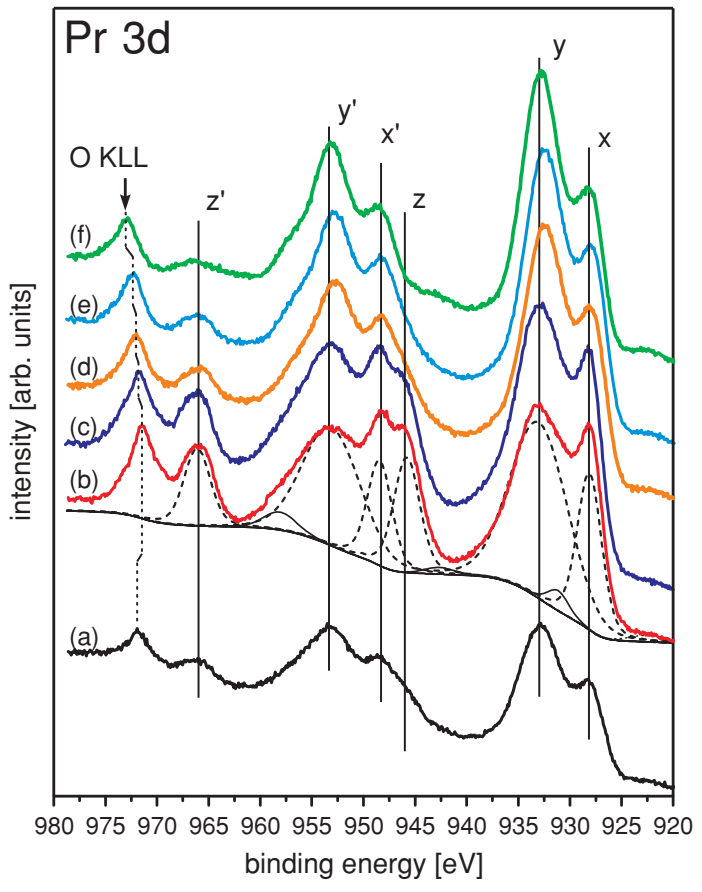

FIG. 3. XP spectra of the Pr $3 d$ core level: (a) untreated, (b) after oxygen plasma treatment (dashed lines represent the single contributions obtained by curve fitting) and after annealing under UHV conditions to (c) 405, (d) 580, (e) 680, and (f) $920 \mathrm{~K}$, respectively. The labels $x, y$, and $z$ denote the assignment to $3 d^{9} 4 f^{3}, 3 d^{9} 4 f^{2}$, and $3 d^{9} 4 f^{1}$ final state configurations, respectively.

this assignment is surprising at first sight since Pr is formally in its highest oxidation state. In fact, neutron scattering studies clearly proved a ground state in which only one $4 f$ electron is localized at the Pr cations. This discrepancy was resolved conceptually on the basis of a hybridization of the $\mathrm{Pr}$ $4 f$ level and the $\mathrm{O} 2 p$ derived valence band. Already localspin-density calculations suggested a difference between valency (tetravalent) and ionicity (between $3+$ and $4+)^{16}$ and confirmed a picture, where one electron is localized at the Pr ion, while further (delocalized) electron density is provided by the $\mathrm{O} 2 p$ band through a $\mathrm{Pr} 4 f-\mathrm{O} 2 p$ hybridization. ${ }^{17}$ Taking this interaction between $\mathrm{O} 2 p$ and $\operatorname{Pr} 4 f$ states and resulting screening options of the $3 d$ hole into account, the complex multitude of peaks in the Pr $3 d$ spectrum can be attributed to three final states, i.e., three different electron configurations of the resulting ion after the core hole generation: The signal with the highest binding energy labeled $z^{\prime}$ can be ascribed to the energetically most unfavorable final state namely a fully unscreened $3 d^{9} 4 f^{1}$ configuration. The corresponding $3 d_{5 / 2}$ emission of the spin-orbit doublet (labeled $z$ in Fig. 3) is found at $\sim 20 \mathrm{eV}$ lower binding energy. The other two signal pairs are due to states where the $3 d$ core hole is screened by additional $f$ electrons originating from the $\mathrm{O} 2 p$ band by virtue of the hybridization with the $f$ states. More precisely, the signals $y / y^{\prime}$ stem from a less screened $3 d^{9} 4 f^{2}$ configuration while signals $x / x^{\prime}$ arise from a highly screened state with an $f^{3}$ occupancy.

Somehow puzzling, however, the XP spectra are very similar to those reported for the sesquioxide $\left(\mathrm{Pr}_{2} \mathrm{O}_{3}\right)$. (Note that for ceria the position and intensity ratios of the photoemission lines shift dramatically upon oxidation. ${ }^{18}$ ) Actually, the $3 d$ emissions of $\operatorname{Pr}_{2} \mathrm{O}_{3}$ are found virtually at the same energies as the $x, x^{\prime}, y$, and $y^{\prime}$ emissions of $\mathrm{PrO}_{2}$. Even some intensity is found at the $z / z^{\prime}$ positions which was explained by valency changes on the surface of the sesquioxide. ${ }^{14}$

All photoemission studies on $\mathrm{PrO}_{2}$ published so far were carried out on samples conventionally prepared. In view of the difficulty to prepare pure and clean $\mathrm{PrO}_{2}$, in particular under UHV conditions, it is an obvious question whether the close similarity between the reported spectra of $\mathrm{PrO}_{2}$ and $\mathrm{Pr}_{2} \mathrm{O}_{3}$ was a result of partially reduced (or contaminated) samples used in former studies. In this case, the existence of signals related to $\mathrm{Pr}^{3+}$ in the $\mathrm{PrO}_{2} 3 d$ spectrum would rather represent a (surface) reduction of the samples than an inherently higher $4 f$ population.

To fully oxidize and clean $\mathrm{PrO}_{2}$ samples for a photoemission study, we subjected praseodymia films to an in situ oxygen plasma treatment directly before the experiments. The Pr $3 d$ signal recorded after the plasma treatment [Fig. 3(b)] clearly shows that previous spectra of $\mathrm{PrO}_{2}$ did not represent stoichiometric but indeed most probably a reduced $\mathrm{PrO}_{2}$. To better illustrate the various contributions, the spectrum was analyzed using a curve fitting routine with a Voigt profile (Fitt 1.2 by Hyun-Jo Kim, Seoul National University). In addition to the three signal pairs (dashed lines) there are small contributions to the spectrum (thin solid line, unlabeled features) which result from the intraatomic multiplet effects as reported by Kotani et al. ${ }^{15}$ and were described by a Gaussian. In comparison to spectrum (a) the differences are obvious: the $3 d^{9}$ $4 f^{1}$ emissions $z$ and $z^{\prime}$ in spectrum (b) are much more intense, representing the highest intensity reported so far. While the large $z$ and $z^{\prime}$ signals indicate a higher abundance of $\mathrm{Pr}^{4+}$ valency, the multitude of the other peaks confirms the relevance of an $\mathrm{O} 2 p$ Pr $4 f$ hybridization also for fully oxidized $\mathrm{PrO}_{2}$ since the emissions labeled $x, x^{\prime}, y$, and $y^{\prime}$ are still present. Compared to spectra of $\operatorname{Pr}_{2} \mathrm{O}_{3}$, however, also differences are observed. In particular, the ratio of the $x$ and $y$ emission intensities is significantly altered. (Also changes in the half-widths are found.) We find good agreement of spectrum (b) with an experimental spectrum obtained for $\mathrm{BaPrO}_{3} \cdot{ }^{19}$ Simulations of this XP spectrum based on calculations for $\mathrm{PrO}_{2}$ yielded an average number of 1.78 electrons for the $\operatorname{Pr} 4 f$ states in the ground state. ${ }^{19}$ Since our experimental results for plasmatreated $\mathrm{PrO}_{2}$ closely resemble the spectra for $\mathrm{BaPrO}_{3}$ we also take this number as an estimate for the average $4 f$ electron number in $\mathrm{PrO}_{2}$ or, to phrase it in the delocalized picture, the degree of $f$ character of the hybridized $4 f-2 p$ level. It is interesting to note that Kotani et al. calculated a $f$ electron occupancy of 1.56 in $\mathrm{PrO}_{2}$ for the ground state based on spectra similar to (a) in Fig. 3, implying that the $f$ character of the hybridized $\operatorname{Pr} 4 f-\mathrm{O} 2 p$ state is apparently more pronounced than believed so far and thus the average $f$-electron count is higher (i.e., in the localized picture, $\mathrm{PrO}_{2}$ would to a larger extent exhibit $\mathrm{Pr}$ in the oxidation state $3+$, i.e., with a $f^{2}$ configuration). The necessary electron density stems from the $\mathrm{O} 2 p$ band. This avoidance of the pure $f^{1}$ configuration and the depopulation of the $\mathrm{O} 2 p$ band could be the reason for the special role of the praseodymia system in the REO series and the easy removability of lattice oxygen. Further theoretical calculations will be necessary to substantiate these conclusions. 
In addition to the plasma treatment the sample was annealed in a stepwise fashion, each step for one minute, to monitor the effect of thermal reduction and corresponding oxygen loss of the oxide surface. Figures 3(c)-3(f) show the effect on the Pr $3 d$ spectra after returning to room temperature. With increasing annealing temperature from 405 to $920 \mathrm{~K}$ the intensity of the signal related to $\operatorname{Pr}^{4+}\left(z, z^{\prime}\right)$ decreases to almost zero. No significant change is observed after heating to $405 \mathrm{~K}$ (spectrum c). However, after heating to $580 \mathrm{~K}$ the $\mathrm{Pr}^{4+}$ emissions $z, z^{\prime}$ together with the emissions $x, x^{\prime}$ considerably decrease in intensity during annealing (spectrum d). The intensity ratio of the $y / x$ and $y^{\prime} / x^{\prime}$ signals, respectively, is now again similar to that of the untreated sample, whereas the width appears to be smaller than in spectrum (a), revealing how sensitive the fully oxidized film is with respect to heating under vacuum conditions. With increasing annealing temperature a gradual reduction of the oxide film and loss of $\mathrm{Pr}^{4+}$ species is observed (spectrum e) up until annealing to $920 \mathrm{~K}$ leads to spectrum (f), which exhibits the typical shape observed for $\mathrm{Pr}_{2} \mathrm{O}_{3}{ }^{7}{ }^{70,12,15}$ The emission from $\mathrm{Pr}^{4+}$ has almost completely vanished. Residual intensity at the position of $z$ is most likely due to surface induced valence changes as mentioned above. LEED shows the fingerprint of a cub$\mathrm{Pr}_{2} \mathrm{O}_{3}(111)$ surface as demonstrated below [cf. Figs. 4(a) and 4(c)]. A change in the $\mathrm{Pr}-\mathrm{O}$ bonding situation is also reflected by the O KLL Auger emission, which shifts to higher binding energy upon heating. A shoulder observed at the higher BE side of signal $y^{\prime}$, now better separated, is due to intraatomic electron-electron multiplet (coulomb) interaction. ${ }^{15}$ These results provide further evidence that spectrum (b) corresponds to the highest oxidized Pr oxide film investigated so far in an UHV environment.

\section{B. TPD of oxygen}

A demonstrating way of observing the oxygen loss of the oxide film upon heating is a TPD experiment with the plasmatreated sample being heated at a constant rate in front of the entrance aperture of a mass spectrometer. The result of such a TPD monitoring of the masses of molecular oxygen, carbon monoxide, and carbon dioxide is shown in Fig. 4(a). No desorption of carbon species is observed over the entire temperature range. This observation is in agreement with a lack of $\mathrm{C} 1 \mathrm{~s}$ emission in XPS which indicates that there is no residual carbon left after the plasma cleaning procedure. As can be seen from the oxygen spectrum, minor desorption of molecular oxygen already starts at temperatures as low as $\sim 373 \mathrm{~K}$. The main desorption signal is observed at $\sim 523 \mathrm{~K}$, followed by two further desorption signals at $\sim 623$ and $\sim 730 \mathrm{~K}$, respectively. These four desorption signals most likely correspond to phase transitions of the oxide film. The Pr $3 d$ XP spectra shown in Figs. 3(c)-3(d) were recorded at temperatures right in between those of the desorption maxima, i.e., the different XP spectra should represent different phases of the oxide film, which phases are still a matter of investigation. The main changes in the Pr $3 d$ spectra are observed after heating the sample above the temperature of the main oxygen desorption signal $(523 \mathrm{~K})$. It is reasonable to assume that the

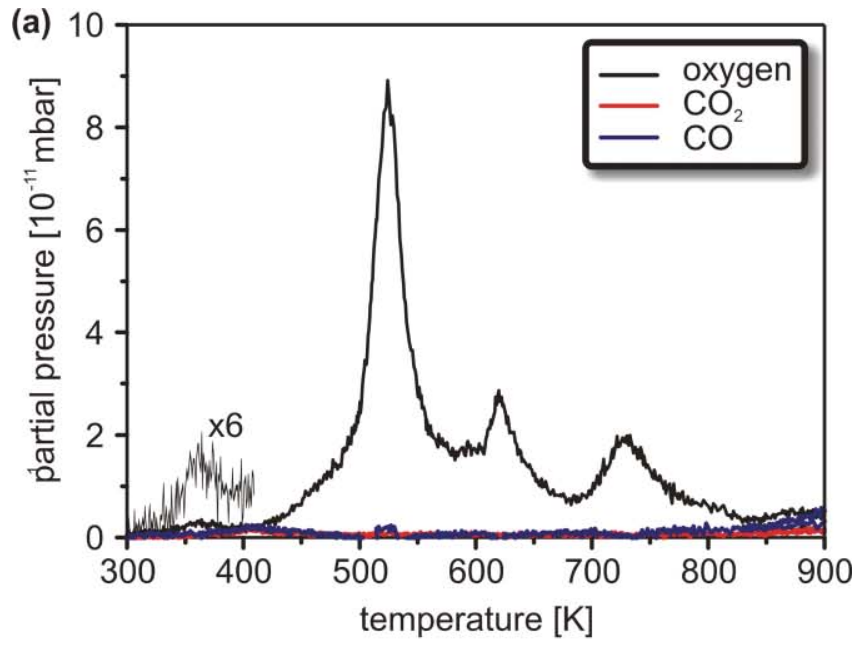

(b)

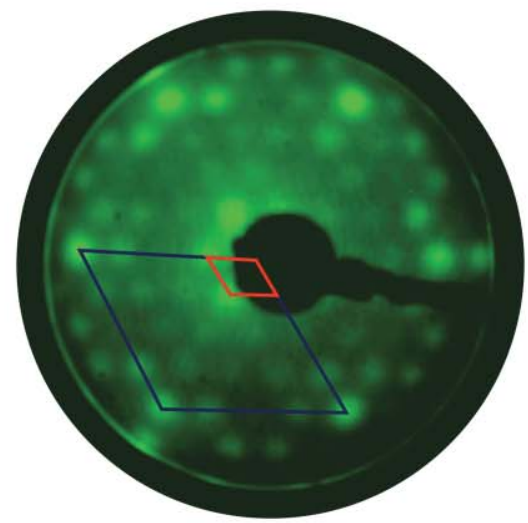

(c)

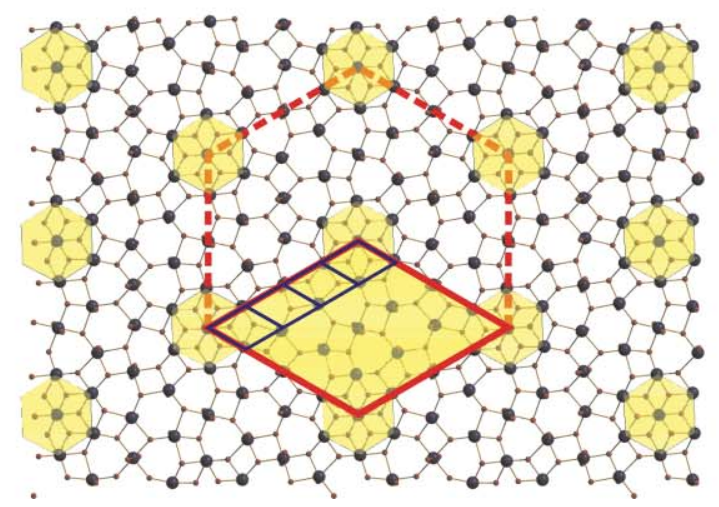

FIG. 4. (a) TPD spectrum of a Pr-oxide film after oxygen plasma treatment depicting the QMS-signal for molecular oxygen, carbon monoxide, and carbon dioxide. No desorbing carbon species, expected to form from residual impurities, is observed to be consistent with the XPS results. Desorption of $\mathrm{O}_{2}$ is observed at several temperatures and with sharp signals most likely corresponding to the transition from one oxide phase to the other. (b) LEED image recorded at $32 \mathrm{eV}$ after heating a plasma-treated film for $30 \mathrm{~min}$ to $950 \mathrm{~K}$. The diffraction pattern represents the cub- $\mathrm{Pr}_{2} \mathrm{O}_{3}$ (111) surface. (c) Schematic view onto the cub- $\mathrm{Pr}_{2} \mathrm{O}_{3}(111)$ plane showing atom positions (cut through bulk crystal). Highlighted areas mark identical structure units. Red and blue diamonds show the unit cells of cub- $\operatorname{Pr}_{2} \mathrm{O}_{3}$ (111) and unreconstructed $\mathrm{Si}(111)$, respectively.

thermodynamically most stable phase of praseodymium oxide under ambient conditions, i.e., $\operatorname{Pr}_{6} \mathrm{O}_{11},{ }^{21,22}$ is then obtained.

When keeping the sample for a longer time $(\sim 30 \mathrm{~min})$ at $900 \mathrm{~K}$ a LEED pattern as shown in Fig. 4(a) is obtained. It is as expected for the (111) surface of a cub- $\operatorname{Pr}_{2} \mathrm{O}_{3}$ film growing lattice-matched with the $\mathrm{Si}(111)$ substrate. The unit cell of 

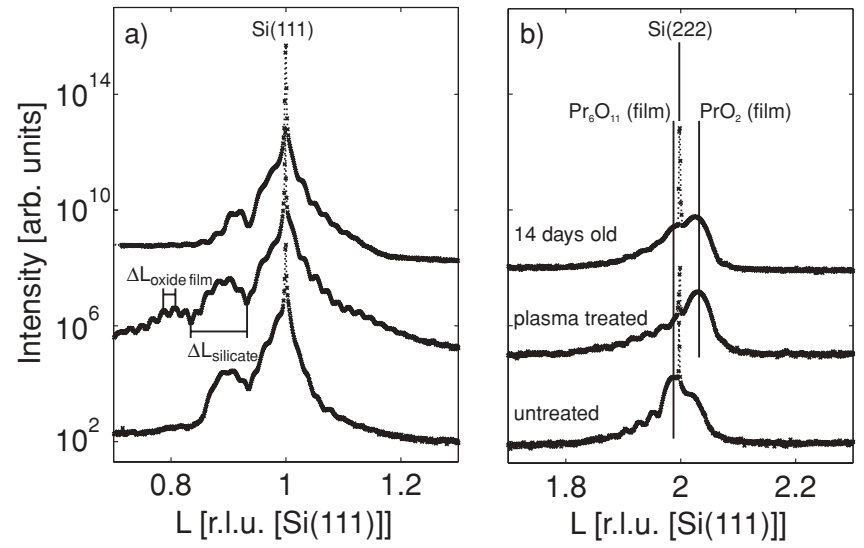

FIG. 5. Si(LLL) XRD measurements close to (a) the Si(111) Bragg peak and (b) the $\mathrm{Si}(222)$ Bragg peak. (a) Fringes due to oxide and silicate are clearly visible while the praseodymia Bragg peaks are veiled from the wings of the strong $\operatorname{Si}(111)$ Bragg peak. The thickness of the praseodymia film and the silicate interface layer can be estimated from $\Delta L_{\text {oxide film }} / d_{\mathrm{Si}}$ and $\Delta L_{\text {silicate }} / d_{\mathrm{Si}}$, respectively. (b) Praseodymia (222) Bragg peaks and oxide film fringes are well resolved while the kinematically forbidden $\operatorname{Si}(222)$ Bragg peak at $L=2$ is very weak.

cub- $\operatorname{Pr}_{2} \mathrm{O}_{3}(111)$ (shown as red diamond in the schematic of Fig. 4(c) is $4 \times 4$ times larger than that of the unreconstructed $\mathrm{Si}(111)$ surface (blue diamonds). Colored areas in Fig. 4(c) are meant as guides to the eye and indicate identical structural units.

The results presented so far show that, by applying an oxygen plasma cleaning procedure, clean, and pure $\mathrm{PrO}_{2}$ films become available for surface science studies in UHV. In combination with annealing in vacuum at low oxygen partial pressure, different phases over the entire range from the dioxide to the sesquioxide become accessible.

\section{X-ray diffraction}

XRD measurements were performed to obtain information about the structural transformations of the praseodymia film during oxygen plasma treatment. Therefore, $\theta-2 \theta$ scans [ $\theta$ indicates the incidence angle with respect to the (111)plane] close to the $\mathrm{Si}(111)$ Bragg peak as well as the $\mathrm{Si}(222)$ Bragg peak were recorded to characterize the structure and morphology of the praseodymia films after different treatments.

As discussed before, ${ }^{8,20,23}$ the diffraction intensity close to the $\mathrm{Si}(111)$ Bragg peak contains the full information about both the vertical lattice constant and the film thickness of the praseodymia films as well as the interface distance and interface roughness due to the interference between substrate and film. Diffraction close to the $\operatorname{Si}(222)$ Bragg peak, however, which is forbidden according to kinematic diffraction theory, is only sensitive to the diffraction from the praseodymia film so that this diffraction condition is better suited to characterize quantitatively the praseodymia film itself and to avoid complications with respect to the interference between film and substrate.

XRD measurements for different praseodymia films close to both the Si(111) Bragg peak and the Si(222) Bragg peak are presented in Figs. 5(a) and 5(b), respectively. Here, we used reciprocal lattice units (r.l.u.), which are scaled to the $\mathrm{Si}$ (111) layer distance $d_{\mathrm{Si}}$, for the vertical scattering vector $\mathbf{Q}=\left(2 \pi / a_{0}\right)(\mathrm{LLL})$ with Si bulk lattice constant $a_{0}=5.4307$ $\AA$ (vertical lattice constant $d_{\mathrm{Si}}=3.1354 \AA$ ).

Figure 5(a) shows the strong and sharp Si(111) Bragg peak for $L=1$ and additional diffraction signals due to the praseodymia film. It is difficult to observe the Bragg peaks of the praseodymia films since they are hidden in the wings of the $\mathrm{Si}(111)$ Bragg peak. Furthermore, fringes due to the finite thickness of praseodymia films (short period in reciprocal space) and silicate interface layers (long period in reciprocal space) are clearly visible. The praseodymia thickness and the silicate layer thickness of 13 and $3 \mathrm{~nm}$, respectively, obtained for the untreated sample are obtained for the plasma-treated film, too. Obviously, the plasma treatment clearly improves the quality of the praseodymia film since the fringes are much better developed. Storage under ambient conditions, however, worsens the quality of the praseodymia films.

The Bragg peaks of the praseodymia films with cubic structure are better visible close to the $\mathrm{Si}(222)$ Bragg peak as shown in Fig. 5(b). Kinematic diffraction theory assumes spherical symmetry of the atomic electron density distribution, and as a consequence the $\mathrm{Si}(222)$ Bragg peak is forbidden. In the experiment, however, it becomes visible due to the nonspherical electron distribution for $\mathrm{Si}$ atoms although the peak intensity is very weak. Thus, the weak but sharp $\operatorname{Si}(222)$ Bragg peak can be used for calibration while interference effects between substrate and film can be neglected since the wings of the $\operatorname{Si}(222)$ Bragg peak rapidly decrease with $L$ values different from $L=2$ due to the semi-infinite Si substrate.

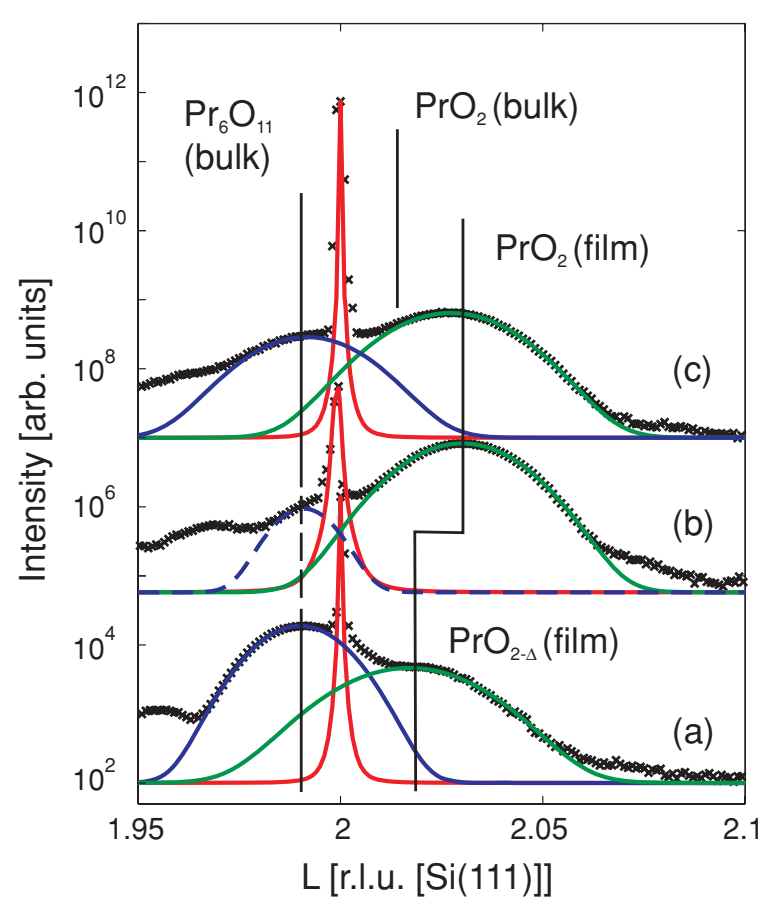

FIG. 6. XRD analysis of the (222) Bragg peaks due to cubic praseodymia films: (a) untreated sample, (b) plasma-treated sample, and (c) after storage under ambient conditions for two weeks. The solid lines are Gaussian fitted to the Bragg peaks of $\operatorname{Pr}_{6} \mathrm{O}_{11}$ (222), $\mathrm{Si}(222), \mathrm{PrO}_{2-\Delta}$ (222), and $\mathrm{PrO}_{2}$ (222). For comparison, Bragg peak positions expected from bulk data reported in literature are presented, too. 
TABLE I. Structural data of the praseodymia films obtained from XRD. The fraction of the $\operatorname{PrO}_{2}$ and $\operatorname{Pr}_{6} \mathrm{O}_{11}$ phases are estimated from the ratio of the corresponding Bragg peak intensities neglecting effects due to the structure factor. The structure factors of both $\operatorname{PrO}_{2}$ and $\operatorname{Pr}_{6} \mathrm{O}_{11}$, however, do not differ drastically since the additional oxygen content of $\mathrm{PrO}_{2}$ (compared to $\left.\operatorname{Pr}_{6} \mathrm{O}_{11}\right)$ attributes only slightly to the structure form factor due to the small atomic form factor of $\mathrm{O}$ compared to $\mathrm{Pr}$.

\begin{tabular}{lccr}
\hline \hline & Untreated & Plasma treated & Stored \\
\hline Vertical lattice constant $\mathrm{PrO}_{2}(\mathrm{pm})$ & 311 & 309 & 309 \\
$\mathrm{PrO}_{2}$ fraction (\%) & 30 & $>95$ & 70 \\
Vertical lattice constant $\mathrm{Pr}_{6} \mathrm{O}_{11}(\mathrm{pm})$ & 315 & $\ldots$ & 315 \\
$\mathrm{Pr}_{6} \mathrm{O}_{11}$ fraction (\%) & 70 & $<5$ & 30 \\
\hline \hline
\end{tabular}

Figure 5(b) shows two Bragg peaks for both the untreated sample and for the sample after exposure to ambient conditions. Therefore, the praseodymia films of these samples split up into different oxide phases as reported previously. $8,12,20$ The plasma-treated sample, however, exhibits only one Bragg peak and, therefore, has only one praseodymia phase. In addition, fringes due to the finite thickness of the praseodymia films are also clearly visible in Fig. 5(b).

The (222) Bragg peaks of the praseodymia films are fitted by Gaussians to determine the vertical lattice constants of the different praseodymia phases as presented in Fig. 6. Both the vertical lattice constants as well as the fractions of the different oxide species, which are estimated from the intensities of the Bragg peaks, are presented in Table I. For the untreated sample, the stronger oxide Bragg peak found at lower $L$ values is attributed to the formation of $\operatorname{Pr}_{6} \mathrm{O}_{11}$ since the vertical lattice parameter of $3.15 \AA$ matches almost the $\operatorname{Pr}_{6} \mathrm{O}_{11}$ bulk value of $3.16 \AA .^{24}$ The second Bragg peak at higher $L$ values is caused by a vertical lattice constant of $3.11 \AA$ matching perfectly the bulk lattice constant of $\mathrm{PrO}_{2}$ reported in literature. ${ }^{24}$

After the plasma treatment, the $\mathrm{PrO}_{2}$ Bragg peak shifts to higher $L$ values. Thus, the vertical lattice constant decreases to $3.09 \AA$ A. Since the bulk lattice constant of cubic praseodymia phases of different stoichiometry decreases as the oxygen content increases ${ }^{25}$ (cf. the smaller lattice constant of $\operatorname{PrO}_{2}$ compared to $\operatorname{Pr}_{6} \mathrm{O}_{11}$ which can formally be conceived as mixture of $\operatorname{PrO}_{2}$ and cubic $\operatorname{Pr}_{2} \mathrm{O}_{3}$ where the latter has a $3.2 \%$ larger lattice constant than $\mathrm{PrO}_{2}$ ) we conclude that the plasma-treated praseodymia film possesses more oxygen than the untreated film. Therefore, the praseodymia phase which has previously been called $\mathrm{PrO}_{2}$ in agreement with literature ${ }^{24}$ exhibits oxygen vacancies and is understoichiometric. Therfore, it has to be called $\mathrm{PrO}_{2-\Delta}$ instead of $\mathrm{PrO}_{2}$ due to the oxygen deficit. Obviously, true $\mathrm{PrO}_{2}$ has only been formed after plasma treatment. This conclusion also explains why in former XPS experiments less intense $\mathrm{PrO}_{2}$ features were observed for the $\operatorname{Pr} 3 d$ photoelectrons and only plasma-treated films show the full $\operatorname{Pr} 3 d$ photoelectron spectrum of stoichiometric $\mathrm{PrO}_{2}$. Our results nicely correspond to the vertical lattice constant of $3.097 \AA$ obtained by recent calculations for $\mathrm{PrO}_{2}$ following the self-interaction corrected local-density approximation, ${ }^{26}$ while XRD experiments reported by the same authors seemed to confirm the experimental value of $3.114 \AA$. Therefore, the discrepancy between theory and experiment has to be attributed to incomplete oxidation of praseodymia while plasma-treated praseodymia films are completely oxidized. Finally, we like to emphasize that the discussion above is based on bulk values while our experimental data are obtained from oxide films. Therefore, the residual discrepancy between the theoretical bulk value and the experimental value for the epitaxial film can be attributed to a slight tetragonal distortion of the praseodymia film and our value for the vertical lattice constant of $\mathrm{PrO}_{2-\Delta}$ film may be larger than the bulk value although it seems to match excellently the bulk value (cf. Fig. 6).

Furthermore, the $\operatorname{Pr}_{6} \mathrm{O}_{11}$ phase within the film almost vanishes during plasma treatment. The intensity oscillations in Fig. 6(a) for smaller $\mathrm{L}$ values can be attributed to fringes of the stoichiometric $\mathrm{PrO}_{2}$ film. Nevertheless, assuming that a $\operatorname{Pr}_{6} \mathrm{O}_{11}$ phase (vertical lattice parameter $3.15 \AA$ ) still exists after the plasma treatment, we fitted a Gaussian at the $\operatorname{Pr}_{6} \mathrm{O}_{11}$ Bragg peak position to the data (dashed line). Thus, the $\operatorname{Pr}_{6} \mathrm{O}_{11}$ content of less than $5 \%$ obtained from this analysis clearly overestimates the $\operatorname{Pr}_{6} \mathrm{O}_{11}$ fraction since we neglect the intensity due to $\mathrm{PrO}_{2}$ fringes. The analysis demonstrates the high efficiency of the plasma treatment to obtain stoichiometric $\mathrm{PrO}_{2}$ films.

After exposure of the plasma-treated film to ambient conditions (at air, room temperature) for 14 days the XRD signal has drastically changed. The $\operatorname{Pr}_{6} \mathrm{O}_{11}$ Bragg peak clearly appears again since $\operatorname{Pr}_{6} \mathrm{O}_{11}$ is the thermodynamically most stable phase under normal conditions. Its intensity, however, is weaker than for the untreated praseodymia film. Thus only smaller parts of the $\mathrm{PrO}_{2}$ film are transformed to $\mathrm{Pr}_{6} \mathrm{O}_{11}$. Furthermore, the vertical lattice constant of the $\mathrm{PrO}_{2}$ phase of $3.09 \AA$ demonstrates that the $\mathrm{PrO}_{2}$ phase is still completely oxidized due to the previous plasma treatment of the praseodymia film.

We like to mention, that in our previously published XRD experiments on praseodymia films oxidized by molecular oxygen at high pressure, $, 12,20$ we observed an oxygendeficient $\mathrm{PrO}_{2-\Delta}$ phase. In the light of the praseodymia films oxidized by plasma this phase of the praseodymia films has clearly to be attributed to $\operatorname{Pr}_{6} \mathrm{O}_{11}$. Furthermore, the new results show that the praseodymia phase formerly declared as stoichiometric $\mathrm{PrO}_{2}$ is oxygen-deficient and has to be called $\mathrm{PrO}_{2-\Delta}$ while completely oxidized $\mathrm{PrO}_{2}$ has been obtained by plasma oxidation.

\section{CONCLUSIONS}

In conclusion, our data indicate that former studies on praseodymia suffered from surface impurities and partially reduced oxide surfaces so that investigations starting from the highest oxidation state were impossible. In contrast, our photoemission and XRD data reveal that a cold RF oxygen plasma treatment of praseodymia films on silicon results in oxide films that are clean, stoichiometrically well defined and of high structural quality. While it is apparently very difficult if not impossible to obtain and maintain the highest oxidized state of $\mathrm{PrO}_{2}$ and a clean surface by other methods, the use of oxygen plasma may also present a viable route for other 
complex oxides. Starting from a clean surface of a complex oxide in its highest oxidation state enables the experimentalist to study in detail all different oxidation states by reducing the oxide in a controlled way under ultrahigh vacuum conditions. In consequence, a plasma cell attached via direct sample transfer to surface science techniques in ultrahigh vacuum opens up unique opportunities for the preparation, processing, and analysis of thin films of complex oxides. Model catalytic studies at different oxygen deficient surfaces prepared via oxygen plasma treatment and annealing in UHV are in progress.

${ }^{1}$ V. E. Henrich and P. A. Cox, The Surface Science of Metal Oxides (Cambridge University Press, Cambridge, 1996).

${ }^{2}$ G. V. Antoshin, K. M. Minachev, and R. V. Dmitriev, Russ. Chem. Bull 16, 1793 (1967)

${ }^{3}$ R. L. Martin, Nature (London) 165, 202 (1950).

${ }^{4}$ T. Schroeder, A. Giussani, H. J. Muessig, G. Weidner, I. Costina, C. Wenger, M. Lukosius, P. Storck, and P. Zaumseil, Microelectron. Eng. 86, 1615 (2009)

${ }^{5}$ S. C. Tsang and C. Bullpit, Sens. Actuators B 52, 226 (1998).

${ }^{6}$ K. Otsuka and M. Kunitomi, J. Catal. 105, 525 (1987).

${ }^{7}$ A. Schaefer, T. Schroeder, G. Lupina, Y. Borchert, J. Dabrowski, C. Wenger, and M. Bäumer, Surf. Sci. 601, 1473 (2007).

${ }^{8}$ T. Weisemoeller, C. Deiter, F. Bertram, S. Gevers, A. Giussani, P. Zaumseil, T. Schroeder, and J. Wollschläger, Appl. Phys. Lett. 93, 032905 (2008).

${ }^{9}$ B. Gehl, U. Leist, V. Aleksandrovic, P. Nickut, V. Zielasek, H. Weller, K. Al-Shamery, and M. Bäumer, Rev. Sci. Instrum. 77, 7 (2006).
${ }^{10}$ S. Lütkehoff, M. Neumann, and A. Slebarski, Phys. Rev. B 52, 13808 (1995).

${ }^{11}$ A. Giussani, O. Seifarth, P. Rodenbach, H. J. Müssig, P. Zaumseil, T. Weisemoller, C. Deiter, J. Wollschläger, P. Storck, and T. Schroeder, J. Appl. Phys. 103, 084110 (2008).

${ }^{12}$ T. Weisemoeller, F. Bertram, S. Gevers, A. Greuling, C. Deiter, H. Tobergte, M. Neumann, J. Wollschläger, A. Giussani, and T. Schroeder, J. Appl. Phys. 105, 124108 (2009).

${ }^{13}$ A. Bianconi, A. Kotani, K. Okada, R. Giorgi, A. Gargano, A. Marcelli, and T. Miyahara, Phys. Rev. B 38, 3433 (1988).

${ }^{14}$ O. Seifarth, J. Dabrowski, P. Zaumseil, S. Müller, D. Schmeißer, H. J. Müssig, and T. Schroeder, J. Vac. Sci. Technol. B 27, 271 (2009).

${ }^{15}$ A. Kotani and H. Ogasawara, J. Electron Spectrosc. Relat. Phenom. 60, 257 (1992)

${ }^{16}$ D. D. Koelling, A. M. Boring, and J. H. Wood, Solid State Commun. 47, 227 (1983).

${ }^{17}$ L. Petit, A. Svane, Z. Szotek, and W. M. Temmerman, Phys. Rev. B 72, 205118 (2005)

${ }^{18}$ D. R. Mullins, S. H. Overbury, and D. R. Huntley, Surf. Sci. 409, 307 (1998).

${ }^{19}$ Z. Hu, G. Kaindl, H. Ogasawara, A. Kotani, and I. Felner, Chem. Phys. Lett. 325, 241 (2000).

${ }^{20}$ S. Gevers, T. Weisemoeller, B. Zimmermann, F. Bertram, C. Deiter, and J. Wollschläger, J. Phys. Condens. Matter 21, 175408 (2009).

${ }^{21}$ G. Adachi and N. Imanaka, Chem. Rev. 98, 1479 (1998).

${ }^{22}$ G. A. M. Hussein, J. Anal. Appl. Pyrolysis 29, 89 (1994).

${ }^{23}$ T. Weisemoeller, F. Bertram, S. Gevers, C. Deiter, A. Greuling, and J. Wollschläger, Phys. Rev. B 79, 245422 (2009).

${ }^{24}$ L. Eyring and N.C. Baenziger, J. Appl. Phys. 33, 428 (1962).

${ }^{25}$ B. G. Hyde, D. J. M. Bevan, and L. Eyring, Philos. Trans. R. Soc. London, Ser. A 259, 583 (1966).

${ }^{26}$ L. Gerward, J. S. Olsen, L. Petit, G. Vaitheeswaran, V. Kanchana, and A. Svane, J. Alloys Compd. 400, 56 (2005). 